\title{
The Clinical Features of Diabetes with Coexisting Autoimmune Thyroid Disease
}

\author{
R. S. Gray, R. Herd and B. F. Clarke \\ Diabetic and Dietetic Department, Royal Infirmary, and Medical Statistics and Computing Unit, \\ University of Edinburgh, Edinburgh, U. K.
}

Summary. A study was made of the clinical features of diabetics with coexisting Graves' disease ( $\mathbf{n}=$ $117)$ or primary hypothyroidism $(\mathrm{n}=98)$. Those with Graves' disease developed thyroid dysfunction and diabetes at an earlier age than patients with primary hypothyroidism. There was, however, no difference between the two groups in respect of sex ratio nor proportion of subjects requiring insulin treatment. In contrast to the general diabetic population, $87 \%$ of diabetics with thyroid disease were female, $56 \%$ required insulin treatment and of patients requiring insulin from diagnosis, the median age at diagnosis of diabetes was 36 years. A strong correlation was observed between age at diagnosis of diabetes and that of hyperthyroidism $(\mathrm{r}=0.71, p<$ $0.001)$ or hypothyroidism ( $\mathrm{r}=0.65, p<0.001$ ). With increasing age at diagnosis of diabetes the interval between diagnosis of diabetes and thyroid disease diminished. The mean \pm SEM interval between diagnosis of diabetes and that of thyroid dysfunction was longer in hypothyroid $(6.7 \pm 1.2$ years) than in hyperthyroid diabetics $(-2.4 \pm 1.2$ years). Neither insulin-dependent nor non-insulin dependent diabetics with associated thyroid disease exhibited a significant seasonal variation in diagnosis or symptomatic onset of diabetes. It is conceivable that where diabetes accompanies autoimmune thyroid disease in the same patient, both conditions may share a common and coincident pathogenesis which is unrelated to acute environmental influences.

Key words: Insulin-dependent diabetes, non-insulin dependent diabetes, autoimmune thyroid disease, Graves' disease, hypothyroidism, diabetic treatment, sex, seasonal variation

It is now recognised that the syndrome of diabetes mellitus has several distinct aetiologies, each resulting in carbohydrate intolerance [1]. A proportion of diabetics suffer concomitantly from autoimmune thyroid disease. In such cases, the aetiology of diabetes is considered by some workers to be itself autoimmune, characteristically leading to insulin dependency [2-4].

We now report on a large group of diabetics with coexisting autoimmune thyroid disease whose clinical characteristics may be compared and contrasted with subjects having diabetes or thyroid disease alone. We also describe a temporal relationship concerning the development of diabetes and thyroid disease in the same individual.

\section{Patients and Methods}

Two hundred and fifteen diabetics with associated thyroid disease were studied. Of these, 117 ( 20 males, 97 females) were hyperthyroid and 98 ( 9 males, 89 females) had primary hypothyroidism. All were attending or had attended the Diabetic and Dietetic Department of the Royal Infirmary, Edinburgh. Where hypothyroidism followed treatment of hyperthyroidism, the patient was classified in the hyperthyroid group only. The clinical diagnoses of hyperthyroidism and primary hypothyroidism were confirmed biochemically by current tests of thyroid function, including estimation of plasma protein-bound iodine, effective thyroxine ratio, total thyroxine and thyrotrophin (TSH) concentrations and by means of the TSH stimulation test. Hyperthyroidism was shown to be due to Graves' disease on the basis of clinical, histological and/or radioisotope scanning evidence whereby patients with an overactive nodular goitre or other cause of non-Graves' hyperthyroidism were excluded. In the population of diabeties under consideration, the aetiology of primary hypothyroidism may be assumed to be autoimmune thyroiditis in the majority of cases, $85 \%$ of whom have high titres of thyroid auto-antibodies [5].

Patients requiring insulin treatment because of weight loss, ketonuria or uncontrolled symptomatic hyperglycaemia, within three months of diagnosis of diabetes were termed "primary" insulin dependent diabetics (IDD). Patients who exhibited secondary sulphonylurea failure requiring insulin therapy more than three months from diagnosis of diabetes were termed "secondary" IDD. Table 1 shows the mean age at diagnosis of diabetes in hyperthyroid and hypothyroid diabetics in relation to diabetic treatment.

For the purpose of examining the seasonal onset of diabetes only, a further 78 patients ( 14 males, 64 females) were considered, 
Table 1. Age at onset of diabetes in relation to diabetic treatment in hyperthyroid and hypothyroid diabetics

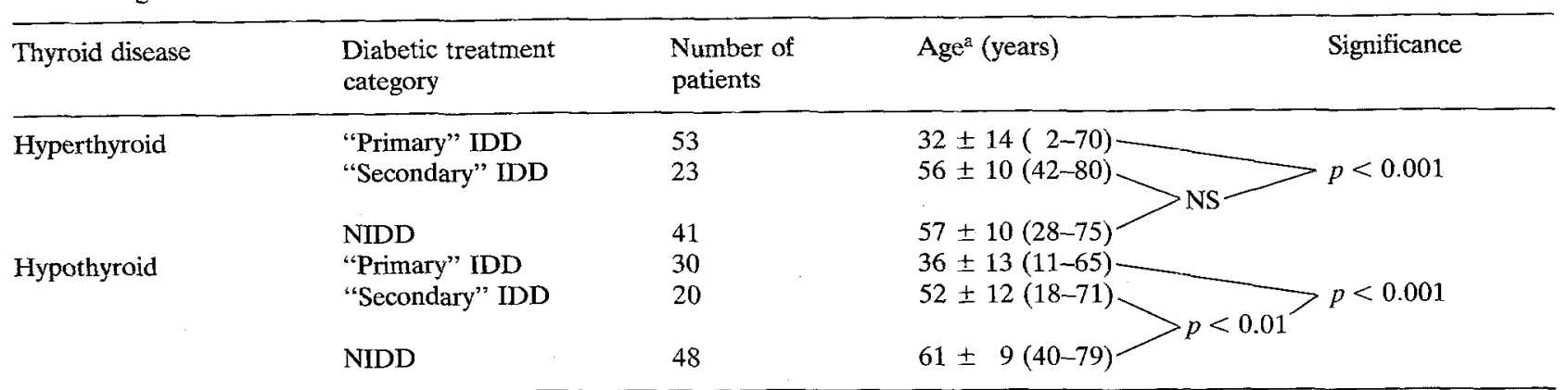

a Expressed as mean $\pm \mathrm{SEM},(+$ range $)$

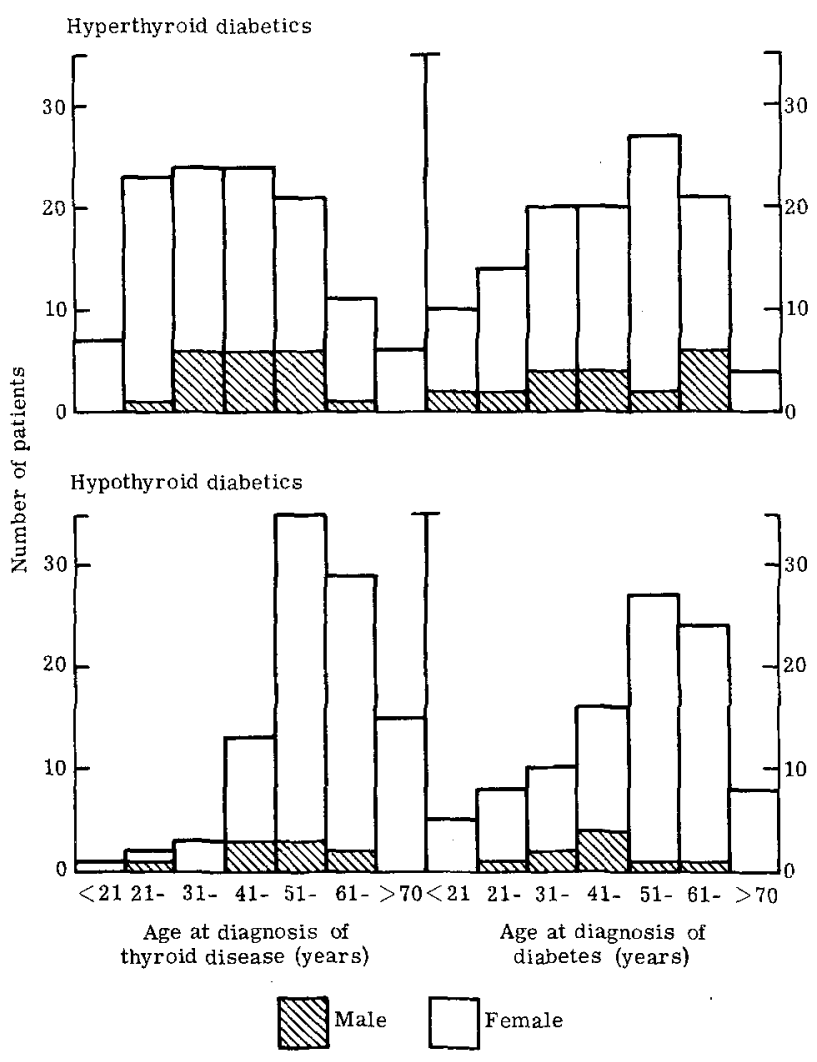

Fig. 1. Distribution of sex and age at diagnosis of diabetes, hyperthyroidism and hypothyroidism in 215 patients studied

who had been found to have subclinical primary thyroid failure as indicated by an elevated plasma TSH concentration $[5,6]$. Their mean \pm standard error (SEM) age at diagnosis of diabetes was $42 \pm 16$ years (range $25-75$ years).

The statistical analysis was carried out using regression techniques employing the GLIM computer package, with the package SPSS being used to extract means and standard errors from the data. Analysis of the seasonal variation in onset of diabetes was made by the test of David and Newell [7].

Results are presented as mean \pm SEM or median + range, where appropriate.

\section{Results}

Figure 1 shows the age at diagnosis of diabetes, hyperthyroidism and hypothyroidism in the patients studied, in relation to their sex distribution. The mean ages at diagnosis of diabetes and thyroid dysfunction in hyperthyroid diabetics ( $46 \pm 2$ and $43 \pm$ 1 years) were younger $(p<0.001 ; p<0.001)$ than those of hypothyroid diabetics $(52 \pm 2$ and $58 \pm 1$ years). There were no significant differences between the sexes in respect of age at diagnosis of these conditions.

Seventy-six (65\%) hyperthyroid and fifty $(51 \%)$ hypothyroid diabetics required insulin treatment. Twenty-eight $(68 \%)$ hyperthyroid and $24(50 \%)$ hypothyroid non-insulin dependent diabetics (NIDD) were treated with a sulphonylurea, the remaining NIDD being treated by diet with or without a biguanide. Table 1 shows that within the diabetic treatment groups of hyperthyroid and hypothyroid diabetics, the "primary" IDD were diagnosed as diabetic at a younger age than the "secondary" IDD combined with NIDD $(p<0.001$, and $p<0.001$ respectively). The median age at diagnosis of diabetes in hyperthyroid and hypothroid "primary" IDD was 36 years. Within the group of hypothyroid, but not hyperthyroid diabetics, the "secondary" IDD were diagnosed as diabetic at a younger age $(p<$ 0.01 ) than the NIDD. In both the hyperthyroid and hypothyroid "secondary" IDD, the mean interval between diagnosis of diabetes and subsequent insulin treatment was $3 \pm 3$ years. The median durations of diabetes in hyperthyroid and hypothyroid NIDD were 7 years $(1-25)$ and 5 years $(1-18)$.

Information regarding the presence of ketonuria at diagnosis of diabetes was available in 82 hyperthyroid and 69 hypothyroid diabetics. Twenty-eight $(34 \%)$ hyperthyroid and $22(32 \%)$ hypothyroid diabetics exhibited ketonuria at diagnosis. When hyperthyroid and hypothyroid diabetics were con- 
Table 2. Interval ${ }^{\mathrm{a}}$ between diagnosis of diabetes and thyroid dysfunction according to age at diagnosis of diabetes

\begin{tabular}{llllllll}
\hline & \multicolumn{7}{c}{ Age at diagnosis of diabetes (years) } \\
\cline { 2 - 7 } & $<21$ & $21-30$ & $31-40$ & $41-50$ & $51-60$ & $61-70$ & $>70$ \\
\hline Hyperthyroid diabetics & $15(1,29)$ & $2(-7,22)$ & $0(-19,30)$ & $-4(-25,7)$ & $-6(-36,17)$ & $-8(-36,6)$ & $-10(-37,0)$ \\
Hypothyroid diabetics & $20(-1,39)$ & $26(-13,40)$ & $18(0,39)$ & $6(-22,19)$ & $3(-8,17)$ & $1(-10,16)$ & $-3(-9,0)$ \\
\hline
\end{tabular}

a Presented as mean + range: where diagnosis of thyroid disease precedes that of diabetes, interval is expressed as negative

A. DIAGNOSIS

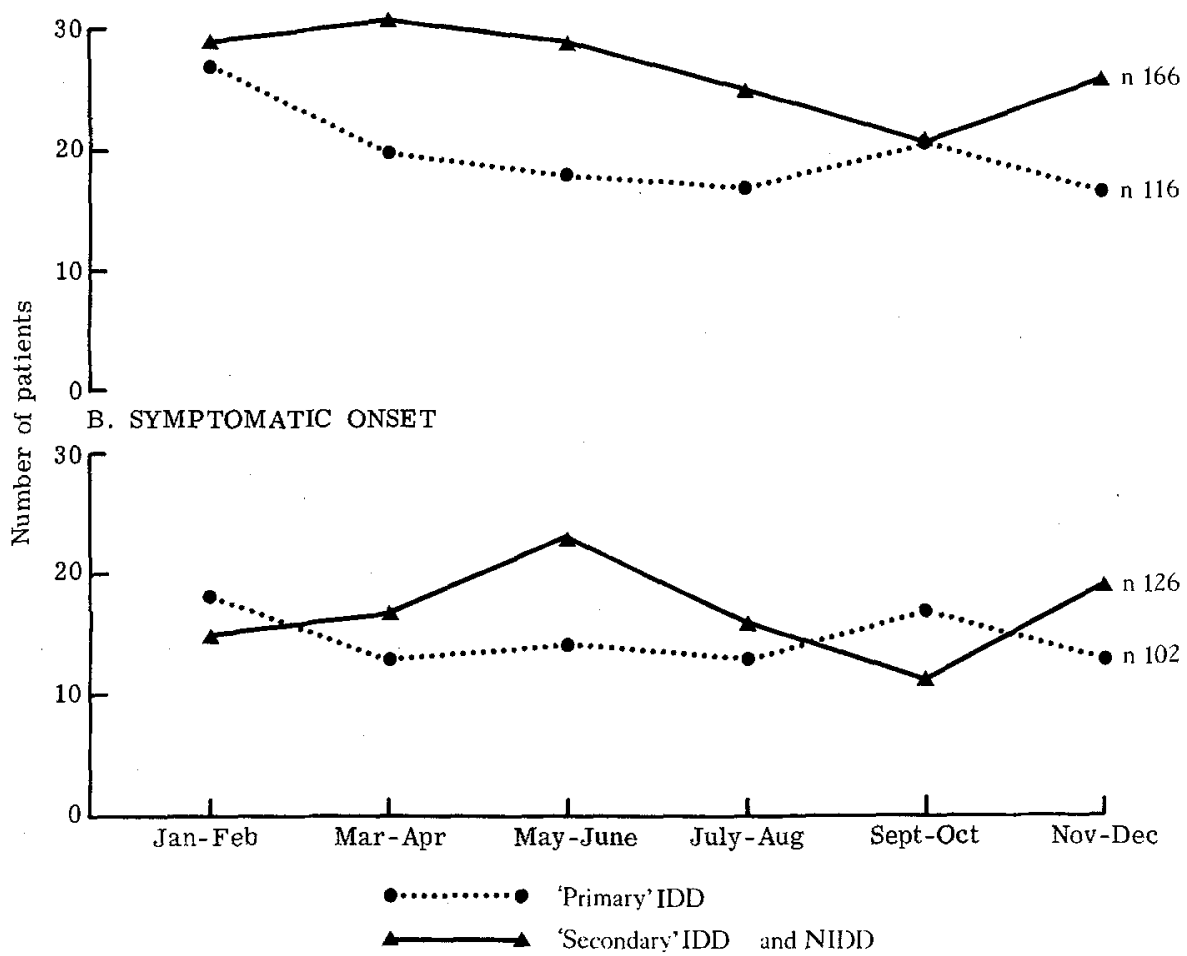

Fig. $2 A$ and $B$. Pattern of seasonal incidence of diagnosis (A) and of symptomatic onset $(B)$ according to diabetic treatment category sidered together, there was a significant inverse correlation $(p<0.001)$ between the incidence of ketonuria and age at diagnosis of diabetes.

A striking correlation was observed between age at diagnosis of diabetes and thyroid dysfunction in hyperthyroid $(\mathrm{r}=0.71, p<0.001)$ and hypothyroid $(\mathrm{r}=0.65, p<0.001)$ diabetics. Similar correlations were found when "primary" IDD $(r=0.62$, $p<0.001 ; \mathbf{r}=0.59, p<0.001$ ), "secondary" IDD $(\mathrm{r}=0.42, p<0.02 ; \mathrm{r}=0.77, p<0.001)$ and NIDD $(\mathrm{r}=0.72, p<0.001 ; \mathrm{r}=0.70, p<0.001)$ were considered independently. A similar correlation ( $\mathrm{r}=0.81, p<0.001)$ was exhibited by hypothyroid NIDD treated by diet with or without a biguanide. For hyperthyroid and hypothyroid diabetics, the regression lines for the three diabetic treatment categories did not differ significantly in slope, when age at onset of diabetes is compared with age at onset of thyroid disease.
Table 2 shows that with increasing age at diagnosis of diabetes the interval between diagnosis of diabetes and that of hyperthyroidism or hypothyroidism fell $(p<0.001$ and $p<0.001)$. This interval was significantly longer $(p<0.001)$ in hypothyroid diabetics $(6.7 \pm 1.2$ years) than in hyperthyroid diabetics $(-2.4 \pm 1.2$ years $)$.

Figure $2 \mathrm{~A}$ shows the seasonal incidence of diagnosis of diabetes in "primary" IDD, and in "secondary" IDD combined with NIDD, whose month of diagnosis of diabetes was recorded. Hyperthyroid and hypothyroid (clinical and subclinical) diabetics were considered together. Figure 2B shows the seasonal incidence of symptomatic onset of diabetes in the same patients, having excluded those whose duration of symptoms exceeded three months or had not been recorded. Neither treatment group showed a significant seasonal variation in incidence of diagnosis nor symptomatic onset of diabetes. 


\section{Discussion}

The Diabetic Department of the Royal Infirmary, Edinburgh, provides care for almost $90 \%$ of the local diabetic population [8] whose female to male ratio is $1.3: 1$ [8]. A third of our general diabetic population is insulin treated [8], the majority of "primary" IDD being diagnosed before the age of 25 years, as reported elsewhere $[9,10]$. In contrast, we now show that diabetics with associated autoimmune thyroid disease exhibit marked female preponderance with a female to male ratio $6.4: 1$. The proportion of insulin dependent to non-insulin dependent diabetics $(1.4: 1)$ is almost three times greater than that observed in our general diabetic population $(1: 2)$. Moreover, the median age at diagnosis of diabetes in "primary" IDD with associated autoimmune thyroid disease is 36 years, that is, appreciably older than in the general diabetic population. Thus, diabetics with associated autoimmune thyroid disease display a number of clinical features at variance with the general diabetic population. On the other hand, both groups of diabetics exhibit an inverse relationship between age at onset and severity of diabetes at presentation. A similar relationship has previously been described in diabetics with associated idiopathic Addison's disease [11] suggesting that the putative autoimmune process responsible for B-cell destruction becomes less aggressive with increasing age. Conflicting reports regarding the prevalence of insulin dependency in diabetics with associated autoimmune thyroid disease may be reconciled on consideration of the age at diagnosis of diabetes. Bottazzo et al. [4] selected patients on the basis of insulin dependence, whose mean age at diagnosis of diabetes was 36 years. Other studies $[12,13]$ which have included late onset diabetics, report a prevalence of insulin dependency to be 43 and $83 \%$ respectively. Our own findings clearly indicate that insulin dependency becomes less prevalent with advancing age at diagnosis of diabetes in those patients having coexisting autoimmune thyroid disease.

It is of interest that the sex ratio and age at diagnosis of autoimmune hyperthyroidism and hypothyroidism are very similar in the diabetic population, reported in this study, and non-diabetic population, reported by others $[12,14]$.

The highly significant correlation between age at diagnosis of diabetes and of thyroid disease is similar to that described in respect of idiopathic Addison's disease and insulin-dependent diabetes [15] and furthers the possibility that these conditions are all caused by a common and coincident pathogenesis. In the present study, we have shown that the correlation between age at diagnosis of diabetes and of thyroid disease is also found in NIDD who presumably share the same aetiology. The correlation extends to diet/ biguanide treated hypothyroid NIDD and cannot, therefore, be attributed to the antithyroid effect of the sulphonylureas [16].

It therefore seems possible that the pathological processes responsible for the development of diabetes and thyroid disease in the same patient are initiated simultaneously. However, the order in which diabetes and hyperthyroidism or hypothyroidism become clinically evident appears to be related to the age at diagnosis of diabetes. Thus, whilst diabetes precedes thyroid disease in juvenile onset diabetics, the order is reversed in late onset diabetics. This observation may be explained by the rapid deterioration of carbohydrate tolerance in juvenile onset diabetics leading quickly to insulin dependency and the early clinical diagnosis of diabetes. Late onset diabetics run a more benign course and thus may have subclinical diabetes for several years prior to its diagnosis. Similarly, the extended natural history of asymptomatic autoimmune thyroiditis $[17,18]$ may explain why the diagnosis of hyperthyroidism precedes that of hypothyroidism, irrespective of the age at diagnosis of diabetes.

Diabetics with associated thyroid disease do not exhibit a seasonal variation in incidence, nor peaks in age at diagnosis of diabetes. There is therefore no epidemiological evidence to incriminate a viral aetiology in the pathogenesis of "autoimmune" diabetes, as has been provided by ourselves [19] and others $[9,20]$ in relation to the more typical predominantly male juvenile onset "primary" IDD.

Evidence derived from histological, immunological, HLA and family studies indicate that Type 1 diabetes and autoimmune thyroid disease are caused by a similar pathogenesis. The present study supports the latter premise and suggests that the pathological processes responsible for the development of diabetes and autoimmune thyroid disease in the same subject are initiated simultaneously, and independently of acute environmental influences.

\section{References}

1. National Diabetes Data Group (1980) Classification and diagnosis of diabetes mellitus and other categories of glucose intolerance. Diabetes 28: 1039-1057

2. Bottazzo GF, Doniach D (1976) Pancreatic autoimmunity and HLA antigens. Lancet 2: 800

3. Irvine WJ (1977) Classification of idiopathic diabetes mellitus. Lancet 1: 638-642

4. Bottazzo GF, Cudworth AG, Moul DJ, Doniach D, Festenstein $H(1978)$ Evidence for a primary autoimmune type of diabetes mellitus. Br Med J 2: 1253-1255

5. Gray RS, Irvine WJ, Toft AD, Seth J, Cameron EHD, Clarke 
BF (1979) Unrecognised thyroid failure in diabetes mellitus. J Clin Lab Immunol 2: 221-224

6. Gray RS, Borsey DO, Seth J, Herd R, Brown NS, Clarke BF (1980) Prevalence of subclinical thyroid failure in insulin dependent diabetes. J Clin Endocrinol Metab 50: 1034-1037

7. David HA, Newell DJ (1965) The identification of annual peak periods for a disease. Biometrics 21: 645-650

8. Falconer DS, Duncan LJP, Smith D (1971) A statistical and genetical study of diabetes. I. Prevalence and morbidity. Ann Hum Genet Lond 34: 347-369

9. Gamble DR, Taylor KW (1969) Seasonal incidence of diabetes mellitus. Br Med J 3: 631-633

10. Gamble DR (1980). An epidemiological study of childhood diabetes affecting two or more siblings. Diabetologia 19: 341-344

11. Gharib H, Gastincau CF (1969) Coexisting Addison's diseasc and diabetes mellitus: Report of 24 cases and review of literature. Mayo Clin Proc 44: 217-227

12. Sugrue D, McEvoy M, Fecly J, Drury MI (1980) Hyperthyroidism in the Land of Graves: Results of treatment by surgery, radio-iodine and carbimazole in 837 cases. Q J Med 49: $51-61$

13. Ganz K, Kozak GP (1974) Diabctes mellitus and primary hypothyroidism. Arch Intern Med 134: 430-432

14. Tunbridge WMG, Evered DC, Hall, R, Appleton D, Brcwis M, Clark F, Grimlcy Evans J, Young E, Bird T, Smith PA (1977) The spectrum of thyroid disease in a community: the Whickham survey. Clin Endocrinol 7: 481-493
15. Irvine WJ, Feek C, Morris PJ (1981) A study of diabetes mellitus in Addison's disease. J Clin I ab Immunol (in press)

16. Hunton RB, Wells MV, Skipper EW (1965) Hypothyroidism in diabetics treated with sulphonylureas. Lancet 2: 155-158

17. Gordin A, Lamberg BA (1975) Natural course of symptomless autoimmune thyroiditis. Lancet 2: 1234-1238

18. Hawkins BR, Cheah PS, Dawkins RL, Whittingham S, Burger HG, Patel Y, Mackay IR, Welborn TA (1980) Diagnostic significance of thyroid microsomal antibodies in a randomly selected population. Lancet 2: 1057-1059

19. Gray RS, Duncan LJP, Clarke BF (1979) Seasonal onset of insulin dependent diabetes in relation to sex and age at onset. Diabetologia 17: 29-32

20. Christau B, Kromann H, Christy R, Anderson OO, Nerup J (1978) Epidemiological studies on the incidence of juvenile onset insulin-dependent diabetes mellitus in Denmark. Diabctologia 15: 224

Received: 20 October 1980

and in revised form: 12 December 1980

Dr. R. S. Gray

Diabetic and Dictetic Department

Royal Infirmary

Edinburgh

Scotland 